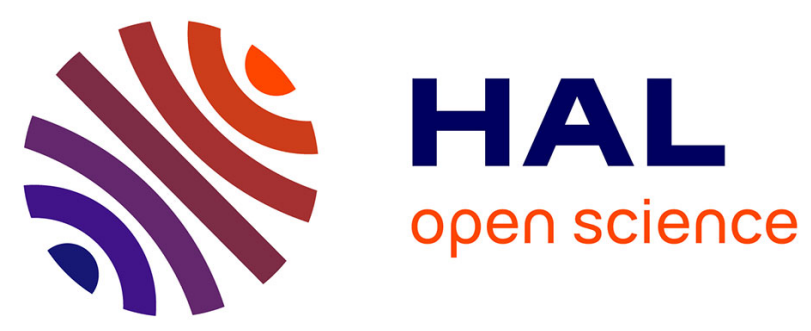

\title{
CNLoc: Channel State Information Assisted Indoor WLAN Localization Using Nomadic Access Points
}

\author{
Jiang Xiao, Huichuwu Li, He Li, Hai Jin
}

\section{To cite this version:}

Jiang Xiao, Huichuwu Li, He Li, Hai Jin. CNLoc: Channel State Information Assisted Indoor WLAN Localization Using Nomadic Access Points. 15th IFIP International Conference on Network and Parallel Computing (NPC), Nov 2018, Muroran, Japan. pp.1-12, 10.1007/978-3-030-05677-3_1. hal-02279560

\section{HAL Id: hal-02279560 \\ https://hal.inria.fr/hal-02279560}

Submitted on 5 Sep 2019

HAL is a multi-disciplinary open access archive for the deposit and dissemination of scientific research documents, whether they are published or not. The documents may come from teaching and research institutions in France or abroad, or from public or private research centers.
L'archive ouverte pluridisciplinaire HAL, est destinée au dépôt et à la diffusion de documents scientifiques de niveau recherche, publiés ou non, émanant des établissements d'enseignement et de recherche français ou étrangers, des laboratoires publics ou privés. 


\title{
CNLoc: Channel State Information Assisted Indoor WLAN Localization Using Nomadic Access Points
}

\author{
Jiang Xiao ${ }^{1}$, Huichuwu $\mathrm{Li}^{1}, \mathrm{He} \mathrm{Li}^{2}$, and Hai $\mathrm{Jin}^{1}$ \\ ${ }^{1}$ Services Computing Technology and System Lab \\ Cluster and Grid Computing Lab \\ School of Computer Science and Technology \\ Huazhong University of Science and Technology, Wuhan, 430074, China \\ ${ }^{2}$ Department of Information and Electronic Engineering \\ Muroran Institute of Technology, Muroran, Hokkaido, Japan \\ jiangxiao@hust.edu.cn
}

\begin{abstract}
Wireless local area network (WLAN) based indoor localization is expanding its fast-paced adoption to facilitate a variety of indoor location-based services (ILBS). Unfortunately, the performance of current WLAN localization systems relying on fixed access points (APs) deployment is constrained by the spatial localizability variance (SLV) problem that different locations may exhibit significantly distinct localization resolution. Prior approaches tackle this problem through nomadic APs with favorable mobility to dynamically adjust the network topology. However, the lack of prior knowledge of nomadic AP's position has been a challenge for location distinction and will lead to prohibitive performance degradation. In this paper, we propose and develop CNLoc, a novel CSI-based (Channel State Information) indoor WLAN localization framework to overcome the location uncertainty of nomadic APs. Our implementation and evaluation show that CNLoc can improve the accuracy with unknown location information of nomadic APs. We also discuss some open issues and new possibilities in future nomadic AP based indoor localization.
\end{abstract}

Keywords: WLAN, CSI, RSS, Mobility.

\section{Introduction}

The rapid proliferation of indoor location-based services (ILBS) has spurred the indoor location market [9], leading to a rash of proposals for developing new localization systems [21]. WLAN-based indoor localization is one of the most efficient methodologies, which applies general WiFi devices in position analysis. Owing to the high availability of infrastructure and low cost, WLAN has become an increasingly attractive choice, ranging from research community [6] [8] [22] to industry (e.g., Google, Apple, Microsoft, etc.). 
In the deployment of WLAN-based indoor localization services, there are some issues which affect the localization accuracy. An important issue is the placement of WLAN access points (APs), which is also very difficult since most APs are deployed for wireless communications. Nomadic APs are those mobile devices that can provide localization services, which brings an opportunity for deployment of WLAN-based indoor localization. In our previous work, we have proposed an indoor localization method based on the nomadic APs, which shows good efficiency in providing localization services.

However, a challenge in the nomadic AP based indoor localization is the location uncertainty of the nomadic APs. In the previous work, we need to know the position of each nomadic AP to estimate the final location. Since the accuracy of the nomadic APs is not always enough and determined, the accumulated error of the estimated location will affect the quality of localization. There are several ways to solve this problem. The first way is to apply some other devices such as microphones or cameras for assistance. Although additional environment information can improve the localization accuracy, the special devices will bring more cost and energy consumption to nomadic APs.

Channel state information (CSI) is another opportunity that improves the localization accuracy by distinguishing the status of different nomadic APs. In the nomadic AP based indoor localization, the location uncertainty is usually brought by moving nomadic APs. Since the mobility of nomadic APs will affect the CSI in WLAN communications, it is possible to distinct static APs and moving APs by analyzing CSI data.

Therefore, in this paper, we propose a new design of CNLoc, to tackle the challenges brought by nomadic APs in indoor WLAN localization. CNLoc leverages the favorable fact that CSI possesses the temporal stability and frequency diversity properties, which makes it capable of inferring the object's status (i.e., moving or static) by the CSI-based location distinction mechanism.

Overall, we summarize the main contributions of our work as:

- We exploit the distinctive capability of CSI to investigate the object's mobility status, which is the crucial premise for better utilizing the nomadic APs' mobility. Due to the advantages of both temporal stability and frequency diversity characteristics, CSI-based location distinction can achieve very high accuracy.

- We overcome the limitation of nomadic APs' location uncertainty by further aggregating the sensor information to the SP-based method which is less sensitive to the nomadic APs' position errors.

- From evaluation results, we observe that CNLoc can achieve great SLV reduction, and outperform the corresponding static AP deployment.

This paper is organized as follows. Section 2 reviews the state-of-the-art researches. Section 3 gives an overview of the technical challenges and then presents the architecture of CNLoc. Section 4 presents our methodology in detail. We present a thorough evaluation in Section 5, and demonstrate that it is more accurate compared to the static AP deployment. In Section 6 we discuss the 
practical issues. Finally, we draw our conclusions and indicate some directions for future work in Section 7.

\section{Related Work}

In this section, we introduce some researches in the following categories: 1) deployment of indoor localization infrastructure, and 2) CSI-assisted localization.

\subsection{Deployment of Indoor Localization Infrastructure}

A localization problem is to transform virtual coordinates of localization infrastructures into physical ones, such as a set of anchors or landmarks. Since the geometric layout of the localization infrastructures significantly affects the localization performance, AP deployment will lead to the SLV problem that the accuracy of indoor localization differs with different layouts. Chen et al. [3] first introduced the landmark placement problem in indoor localization with wireless networks and proposed a placement algorithm to minimize the maximum localization error.

Dulman et al. [4] focused on the anchor deployment in wireless networks. Meng et al. [10] proposed an optimal AP deployment method to improve positioning accuracy in indoor Wi-Fi environments, which maximizes the RSS (radio signal strength) euclidean distance between physical locations.

Due to the complex indoor structure, AP deployment inevitably incurs the SLV problem, where the localization accuracy differs at different locations, leading to user experience inconsistence. Lin et al. [7] proposed an AP selection mechanism based on AP positioning capabilities to improve WiFi fingerprinting accuracy. Gao et al. [5] optimized the placement of landmarks for localization in a warehouse by maximizing the difference degree in each space unit.

\subsection{CSI-assisted Localization}

The accuracy of RSS-based indoor localization systems is limited by the multipath effect $[1,24]$. Bhartia et al. [2] measured the frequency diversity of WLAN channels and proposed some methods to harness diversity by leveraging the CSI in WLAN communications. Yang et al. [23] first introduced the CSI into WLAN-based indoor localization systems and analyzed the frequency diversity from collected CSI data. Wu et al. [18] proposed FILA which is a novel approach that eliminates the multipath effect by leveraging the CSI in indoor scenarios. The CSI is also applied to separate line-of-sight (LOS) path in communications, which can assist location estimation in complex indoor environments $[14,15]$.

Building location fingerprints is another way to optimize the multipath effect in indoor WLAN localization. Sen et al. [16] proposed an indoor localization system called PinLoc that builds location signatures by harnessing the CSI in WLAN channels. Moreover, since many works focused on the motion detection by using WLAN CSI, it is possible to detect the object position directly with 


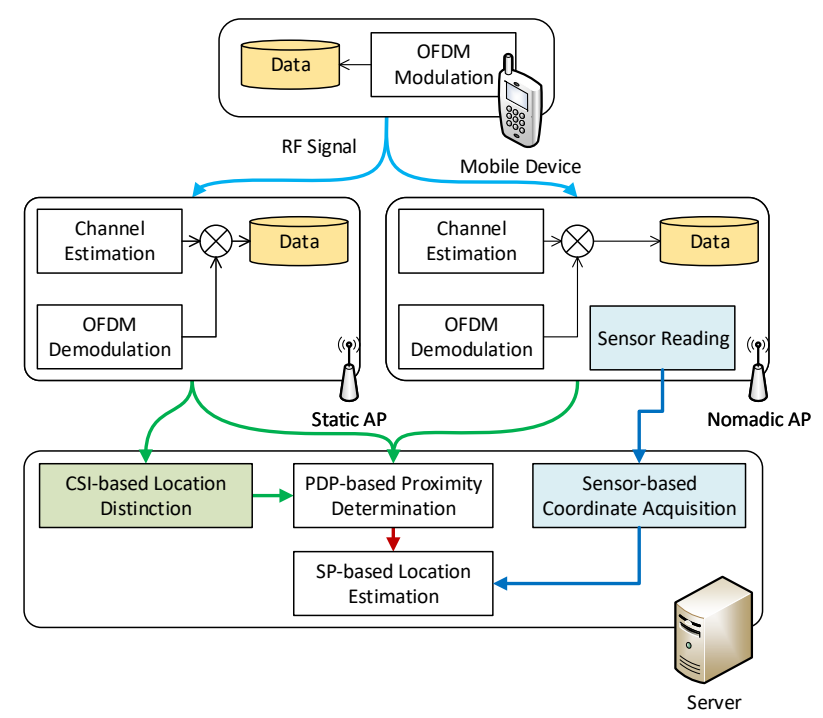

Fig. 1. Architecture of CNLoc

the similar method. Pilot is a device-free indoor localization system that builds different radio maps with CSI data to estimate the positions of entities in the WLAN signal area [19].

\section{CNLoc Framework}

To deal with the SLV problem due to the static deployment of APs, we leverage nomadic APs to improve localization accuracy and mitigate user experience inconsistence at different locations. This section starts with the challenges to utilize nomadic APs for localization, before presenting the overall framework of our CNLoc system.

To harness nomadic APs to establish a dynamic topology so as to avoid the SLV problem, we need to address the following challenge: how to resolve the location uncertainty of nomadic APs for robust location determination? Since nomadic APs tend to move stochastically within the area of interest, it is difficult to obtain their coordinates accurately. As the location estimation error of nomadic APs accumulates, the localization accuracy also degrades. To deal with this challenge, we design a localization framework that is less sensitive to the location uncertainty of nomadic APs.

We harness the mobility of nomadic APs via space partition. In this way, CNLoc neither requires a pre-collected fingerprint database, which is laborintensive to construct [24], nor sophisticated calibration to achieve accurate ranging in complex indoor environments [18]. Figure 1 shows the iterative localization framework, which is resilient to location uncertainty of nomadic APs 
and gradually converges to an accurate location estimate. To enable proximity determination in multipath and NLOS environments, we introduce CSI into the time domain and adopt PDP (power of direct path) for distance estimates. PDP filters out signal power from paths with long delays so as to mitigate the impact of multipath on distance estimation. Since CNLoc only needs relative proximity rather than precise distance between each nomadic AP and the object, the adoption of PDP is sufficient to achieve high localization accuracy even in complex indoor environments. Different from the preliminary work NomLoc [20], we do not assume the coordinates of nomadic APs are known, and obtain them using sensor-based coordinate acquisition.

\section{Methodology}

This section presents the detailed design of the three key modules, 1) PDP-based proximity determination, 2) CSI-based location distinction, and 3) Sensor-based coordinate acquisition.

\subsection{Proximity Determination}

CNLoc uses the maximum power of the power delay profile to approximate PDP of links if there is a strong LOS path. However, it may over-estimate the distance if the LOS path is severely attenuated or even blocked in NLOS propagation situations. To mitigate the impact of NLOS propagation or propagation without a LOS path, CNLoc adopts previous approaches on CSI-based LOS identification schemes for both nomadic and static APs with at least two antennas [17]. As such, we use the PDP as an indicator for proximity determination, which filters out signal power from paths with long delays. Note that CNLoc may still falsely determine the proximity information if there is no LOS path. Nevertheless, since we formulate the localization problem into a space partition problem, which is solved by optimization with redundant measurements, our approach can also tolerate certain extent of errors induced by NLOS propagation.

\subsection{CSI-based Location Distinction}

In current CNLoc design, SP-based algorithm is operated under the assumption that the object stays at an identical location. In other words, the status of the object is necessitated to be stationary during the positioning procedure. In this case, we can derive the object's location jointly from the partition results of nomadic APs. It is unlikely to directly apply the proposed SP-based derivation using the measurement of the nomadic AP at multiple positions if the object is walking around in an indoor venue. For example, if an object moves from location $\mathrm{A}\left(L_{A}\right)$ to location $\mathrm{B}\left(L_{B}\right)$, it is inappropriate to aggregate the SPbased output of $L_{B}$ with $L_{A}$ for positioning. This is because the present partition result of $L_{B}$ only correlates with the preceding ones at $L_{B}$, while independent of those at $L_{A}$. As a consequence, it raises a prerequisite of detecting the mobility 
status of the object, i.e., static or moving, which directly bounds up with the outcome of SP-based algorithm. Now that we need to take the object's status into consideration in the design of CNLoc. Because it needs to be guaranteed that the object keeps stationary as long as the nomadic APs fulfil the localization task. To achieve this, we focus our efforts on the location distinction relying on the fine-grained CSI. The basic idea is to exploit the suitable CSI-based feature which can distinguish the statuses between static and moving, taking advantage of both the temporal stability and frequency diversity characteristics. We denote the CSI measurements over sliding window $W$ of length $N$ by $\mathbb{H}$ as,

$$
\mathbb{H}=\left[\mathrm{H}_{1}, \mathrm{H}_{2}, \ldots, \mathrm{H}_{N}\right]
$$

For each $\mathrm{H}_{i}$, it consists of 30 subcarriers and can be expressed as a vector $\mathrm{H}_{i}$,

$$
\mathrm{H}_{i}=\left[\left|H_{i}^{1}\right|,\left|H_{i}^{2}\right|, \vdots,\left|H_{i}^{30}\right|\right]^{T}
$$

where $\left|H_{i}^{k}\right|$ corresponds to the amplitude of $k$-th subcarrier CSI.

The location distinction feature can then be formulated as the following $\mathbf{C}_{\mathbf{t}}$ :

$$
\mathbf{C}_{\mathbf{t}}=\frac{1}{N-1} \sum_{j=1}^{N} \operatorname{corr}\left(\mathrm{H}_{t}, \mathrm{H}_{t-j}\right)
$$

In CNLoc, $\mathbf{C}_{\mathbf{t}}$ is compared to a preset threshold $\tau$. If $\mathbf{C}_{\mathbf{t}}$ is a higher value than the $\tau$, the object is determined to stay stationary in the area of interest without tendency to change position. On the other hand, the movement of the object will be detected when $\mathbf{C}_{\mathbf{t}}$ falls below the $\tau$. Moreover, we can fuse the detection outputs over multiple links to produce a more accurate result.

To summarize, we can employ the CSI-based location distinction feature from multiple static APs deployed in the positioning region. Lying on the benefits of temporal stability and frequency diversity, such feature can be steady in static status while sensitive to the mobility of the object.

\subsection{Sensor-based Coordinate Acquisition}

As previously noted in Section 3, the uncertainty of nomadic APs' location can result in performance degradation, which is incompatible with our design objective. To confront such difficulty, we assume built-in sensors of nomadic APs become handy tools for identifying the absolute coordinates. In particular, when the prior knowledge of nomadic APs' initial coordinate is available, we suggest a simple yet effective dead reckoning approach based on the sensor information including three phases: 1) leveraging low-pass filter and additional constrains comparing with existing methods for step detection, 2) minimizing the efforts for personalize step length estimation, and 3) direction determination. This method makes it very convenient to ensure accurate nomadic APs' coordinates for optimal preparation of SP-based location estimation. 
We start by detecting and counting the number of steps during nomadic APs' movement using the accelerometer sensor reading. A novel AFSM (augment finite state machine) algorithm is derived to achieve this goal. AFSM algorithm incorporates the following functionalities:

- to apply the butterworth low pass filter for mitigating the high frequency noise and spikes in raw acceleration magnitudes as to recover the true periodicity of the steps;

- to further remove the erroneous detection by adding two-fold heuristic constrains: 1) for each step, the time duration in respect to the descend and ascend parts of vertical acceleration should be identical; and 2) the maximum time duration of one step is limited.

As the second step, we directly apply the well-known step length model [12] [13] to estimate the step length $a$ as follows,

$$
l=a * f+b
$$

where $a, b$ are the parameters need to be estimated, $f$ is the frequency of steps. More specifically, we eliminate the time-consuming calibration efforts with a personalized estimation method. In our experiment, we observe that the nomadic APs with similar variance and average of the vertical acceleration in one step are likely to exhibit similar step length. Relying on this fact, we modify the model for each nomadic AP with minimal efforts required from it. For instance, when a new nomadic AP is tracked for the first step, the model computes the similarly in terms of feature parameters including acceleration variance $V$, mean $M$, and frequency $f$ between this new comer and the previous nomadic APs. If it shares the same feature as that of the previous nomadic AP, they presumably share the same $a, b$. Direction of the nomadic APs' movement is another key factor for acquiring the coordinates. In complex indoor environments, the accuracy of direction determination can be influenced by both ferromagnetic and electrical materials in the vicinity. Fortunately, the gyroscope is decoupled from the geomagnetic sensor, which is insensitive to ambient magnetic fields. Therefore, CNLoc leverages the magnetometer to provide the initial phase as well the gyroscope sensor to obtain the relative angular displacement of the nomadic AP for the purpose of direction determination. This means that CNLoc can detect the orientation of nomadic APs' movement even in the face of the surrounding noises.

\section{Performance Evaluation}

We evaluate the performance of CNLoc system in this section. We collect CSI measurements from external NICs and sensor readings from smartphones because CSI information is currently unavailable on phones. Each nomadic AP is placed on a wheeled desk and pushed by volunteers to move randomly in the area of interest. To record the ground truth trajectories of each nomadic AP, we record the experiments via video and adopt computer vision based localization scheme to pinpoint the location of each nomadic AP. 


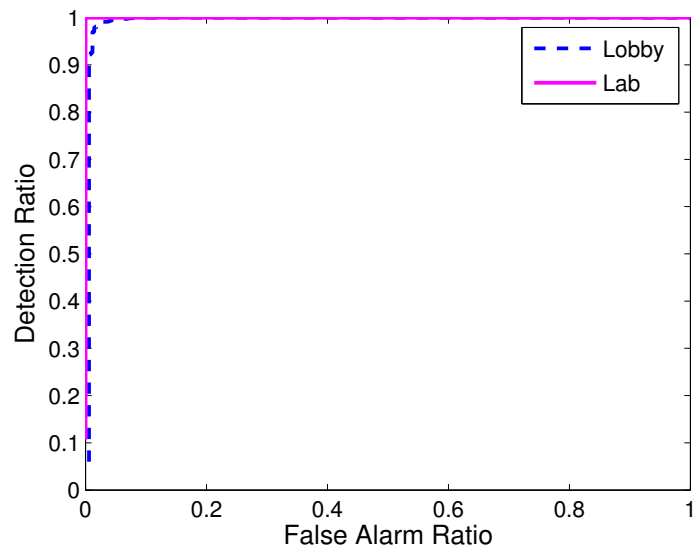

Fig. 2. Location Distinction Accuracy

\subsection{Performance of CSI-based Location Distinction}

Next comes to study the performance of location distinction based on CSI in the scenarios Lab and Lobby. In Lab, we first keep the object staying at site $L_{2}$ for CSI measurements. Afterwards, the object slightly moves to a close site with around $1 m$ distance. By leveraging the CSI-based location distinction feature, we can identify whether the object has changed the location. Figure 2 depicts the results in terms of detection rate (Y-axis) versus false alarm (X-axis). As shown in Figure 2, the FP rate is negligible which proves the effectiveness of the proposed approach in Section 5.1. We further perform the similar measurements at sites $L_{5}$ and a position one meter nearby in Lobby. Even in such a considerably large area, the FP rate only increases very slightly. Hence, we show that in practice, the CSI-based location distinction technique makes it effective and reliable enough to imply the mobility status of the object.

\subsection{Impact of Nomadic APs' Location Uncertainty}

Finally, to provide insights into how the uncertainty of nomadic APs' location influences the overall performance, we show the results in Figure 3(a) and 3(b). We can observe in Figure 3(a) that the sensor information is responsive enough to handle such uncertainty. In general, the smaller coordinate error improves the performance of location estimation in Lab due to the SP-based method barely depends on the AP location which other range-based methods do. We also obtain similar results in Lobby as shown in Figure 3(b). Thus, it demonstrates the promise of ensuring the overall localization performance by accurately coordinate acquisition with embedded sensors of nomadic APs. 


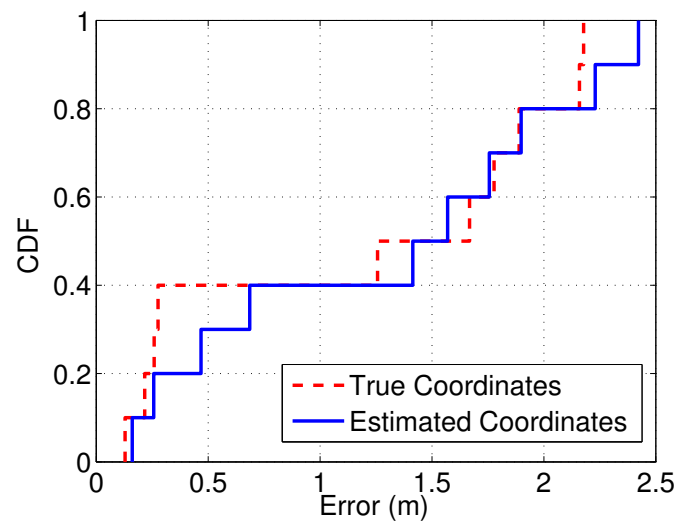

(a) Lab

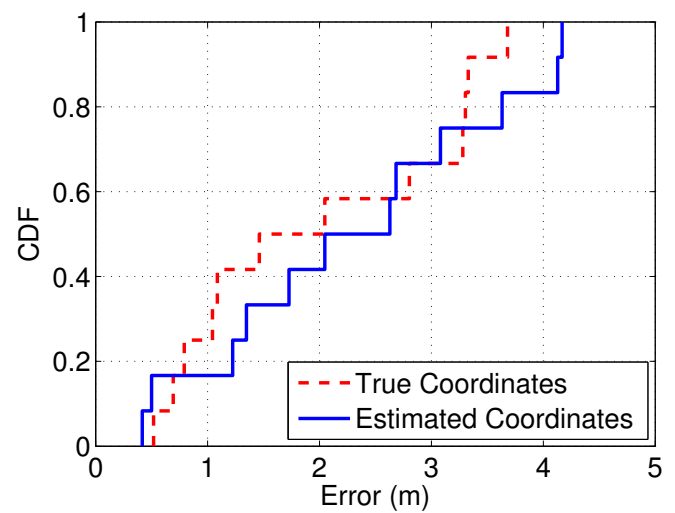

(b) Lobby

Fig. 3. Sensor-based Coordinate Acquisition Performance in Two Scenarios

\section{Discussion}

As an important step forward, CNLoc leaves several open issues and new possibilities for future research. In this section, we briefly comment on the most of important of these here.

\subsection{Influence of AP Mobility on CSI Measurement}

A possible side-effect of nomadic APs lies in the fact that the dynamic movement can give rise to Doppler shift. In particular, nomadic APs move relatively towards or away from an object, resulting in positive or negative Doppler shift as transmission frequency changed. Such Doppler shift may bring in undesirable impact on CSI measurement and degrade the performance of SP-based algorithm. 
We then calculate the Doppler shift frequency $\Delta f$ as below:

$$
\Delta f=\frac{v_{\text {nom } A P}}{v_{\text {nom } A P}+c} f_{0}
$$

where $c$ is the speed of light (i.e., $3 \times 10^{8} \mathrm{~m} / \mathrm{sec}$ ), $v_{\text {nom } A P}$ and $f_{0}$ are the speed and frequency of a nomadic AP, respectively. In the typical $2.4 \mathrm{GHz}$ wireless networks, a moving nomadic AP with velocity of $v_{\text {nomAP }}$ (i.e., $2 \mathrm{~m} / \mathrm{sec}$ ) results in a maximum Doppler shift of $2 \Delta f=32 \mathrm{~Hz}$ which is very small comparing to the center frequency and can be negligible [11].

\subsection{Impact of Diverse APs}

Note that CSI is the key to enable proximity determination and location distinction, which is collected at the AP side in our system. All the APs in current prototype are identical. In practice, the WLAN-based localization infrastructure can be consisted of a variety of APs supplied by different manufacturers (e.g., Belkin, D-Link, Linksys). Yet these APs can have diverse antenna gain which is an influential factor for proximity determination. To handle this factor, we suggest that multiple APs mutually measure the transmission power and then proceed to server for calibrating the differences.

\subsection{Improvement with Nomadic APs' Moving Pattern}

From our evaluation results, CNLoc is capable of improving AP deployment by performing random walk of nomadic APs. Nevertheless, it still leaves upside potential for specifying moving pattern to cover the region of poor localizability. Intuitively, the more the moving traces of nomadic APs approaching an area of dissatisfied localizability, the higher the effectiveness of SP-based scheme. That means optimizing moving pattern can lead to optimum coverage of AP deployment, which ensures to provide users better experience of ILBS at any indoor locations. To this end, we are interested in studying such influence on localization performance resulting from moving pattern of nomadic APs in the future.

\section{Conclusion}

CSI in WLAN communications provides vast opportunities for indoor localization. With the help of fine-grained CSI data, the CNLoc framework advocates the use of mobility for addressing the critical SLV problem, and improves the accuracy of the nomadic AP based localization. Our approach is, to the best of our knowledge, the first one to investigate the static AP deployment, and harness the mobility of nomadic APs to adjust the network topology. To be specific, in the mobile environment, CNLoc also shows good performance in monitoring the object's mobility status without calibration efforts. Furthermore, it permits the sensor-based information for resolving the location uncertainty of nomadic 
APs'. Through extensive experiments, we show the benefits of CNLoc in effectively reducing the SLV and improving the localization accuracy compared to static AP deployment.

In summary, we have taken an important first step towards enabling the mobility of APs for indoor positioning. Our major ongoing work is to arrogate multiple nomadic APs for overall performance enhancement.

\section{Acknowledgment}

This work is supported by National Science Foundation of China under Grant No. 61702203, Hubei Provincial Natural Science Foundation General Program No. 2018CFB133.

\section{References}

1. Bahl, P., Padmanabhan, V.N.: Radar: an in-building RF-based user location and tracking system. In: Proceedings of 2000 IEEE Conference on Computer Communications (INFOCOM). vol. 2, pp. 775-784 (2000)

2. Bhartia, A., Chen, Y.C., Rallapalli, S., Qiu, L.: Harnessing frequency diversity in WiFi networks. In: Proceedings of the 17th Annual International Conference on Mobile Computing and Networking (MobiCom). pp. 253-264. ACM, New York, NY, USA (2011)

3. Chen, Y., Francisco, J.A., Trappe, W., Martin, R.P.: A practical approach to landmark deployment for indoor localization. In: Proceedings of the 6th Annual IEEE Communications Society Conference on Sensor, Mesh and Ad Hoc Communications and Networks (SECON). vol. 1, pp. 365-373 (2006)

4. Dulman, S.O., Baggio, A., Havinga, P.J., Langendoen, K.G.: A geometrical perspective on localization. In: Proceedings of the first ACM international workshop on Mobile entity localization and tracking in GPS-less environments (MELT). pp. 85-90. ACM, New York, NY, USA (2008)

5. Gao, X., Wang, J., Chen, W.: Land-mark placement for reliable localization of automatic guided vehicle in warehouse environment. In: Proceedings of 2017 IEEE International Conference on Robotics and Biomimetics (ROBIO). pp. 1900-1905 (2015)

6. Gu, Y., Lo, A., Niemegeers, I.: A survey of indoor positioning systems for wireless personal networks. IEEE Communications Surveys \& Tutorials (COMST) 11(1), 13-32 (2009)

7. Lin, T.N., Fang, S.H., Tseng, W.H., Lee, C.W., Hsieh, J.W.: A groupdiscrimination-based access point selection for WLAN fingerprinting localization. IEEE Transactions on Vehicular Technology (TVT) 63(8), 3967-3976 (2014)

8. Liu, H., Darabi, H., Banerjee, P., Liu, J.: Survey of wireless indoor positioning techniques and systems. IEEE Transactions on Systems, Man, and Cybernetics (TSMC), Part C (Applications and Reviews) 37(6), 1067-1080 (2007)

9. marketsandmarkets.com: Indoor location market by component. http://www.marketsandmarkets.com/Market-Reports/ indoor-positioning-navigation-ipin-market-989.html (2017) 
10. Meng, W., He, Y., Deng, Z., Li, C.: Optimized access points deployment for WLAN indoor positioning system. In: Proceedings of 2012 IEEE Wireless Communications and Networking Conference (WCNC). pp. 2457-2461 (2012)

11. Pu, Q., Gupta, S., Gollakota, S., Patel, S.: Whole-home gesture recognition using wireless signals. In: Proceedings of the 19th Annual International Conference on Mobile Computing and Networking (MobiCom). pp. 27-38. ACM, New York, NY, USA (2013)

12. Rai, A., Chintalapudi, K.K., Padmanabhan, V.N., Sen, R.: Zee: Zero-effort crowdsourcing for indoor localization. In: Proceedings of the 18th Annual International Conference on Mobile Computing and Networking (MobiCom). pp. 293-304. ACM, New York, NY, USA (2012)

13. Ramirez, L., Dyrks, T., Gerwinski, J., Betz, M., Scholz, M., Wulf, V.: Landmarke: An ad hoc deployable ubicomp infrastructure to support indoor navigation of firefighters. Personal Ubiquitous Computing (PUC) 16(8), 1025-1038 (2012)

14. Sen, S., Choudhury, R.R., Nelakuditi, S.: Spinloc: Spin once to know your location. In: Proceedings of the 13th Workshop on Mobile Computing Systems and Applications (HotMobile). pp. 12:1-12:6. ACM, New York, NY, USA (2012)

15. Sen, S., Lee, J., Kim, K.H., Congdon, P.: Avoiding multipath to revive inbuilding WiFi localization. In: Proceeding of the 11th International Conference on Mobile Systems, Applications, and Services (MobiSys). pp. 249-262. ACM, New York, NY, USA (2013)

16. Sen, S., Radunovic, B., Choudhury, R.R., Minka, T.: You are facing the mona lisa: Spot localization using PHY layer information. In: Proceedings of the 10th International Conference on Mobile Systems, Applications, and Services (MobiSys). pp. 183-196. ACM, New York, NY, USA (2012)

17. Wu, C., Yang, Z., Zhou, Z., Qian, K., Liu, Y., Liu, M.: Phaseu: Real-time LOS identification with WiFi. In: Proceedings of 2015 IEEE Conference on Computer Communications (INFOCOM). pp. 2038-2046 (2015)

18. Wu, K., Xiao, J., Yi, Y., Gao, M., Ni, L.M.: Fila: Fine-grained indoor localization. In: Proceedings of 2012 IEEE Conference on Computer Communications (INFOCOM). pp. 2210-2218 (2012)

19. Xiao, J., Wu, K., Yi, Y., Wang, L., Ni, L.M.: Pilot: Passive device-free indoor localization using channel state information. In: Proceedings of 2013 IEEE 33rd International Conference on Distributed Computing Systems (ICDCS). pp. 236$245(2013)$

20. Xiao, J., Yi, Y., Wang, L., Li, H., Zhou, Z., Wu, K., Ni, L.M.: Nomloc: Calibrationfree indoor localization with nomadic access points. In: Proceedings of 2014 IEEE 34th International Conference on Distributed Computing Systems (ICDCS). pp. 587-596 (2014)

21. Xiao, J., Zhou, Z., Yi, Y., Ni, L.M.: A survey on wireless indoor localization from the device perspective. ACM Computing Surveys (CSUR) 49(2), 25:1-25:31 (2016)

22. Yang, Z., Wu, C., Zhou, Z., Zhang, X., Wang, X., Liu, Y.: Mobility increases localizability: A survey on wireless indoor localization using inertial sensors. ACM Computing Surveys (CSUR) 47(3), 54:1-54:34 (2015)

23. Yang, Z., Zhou, Z., Liu, Y.: From RSSI to CSI: Indoor localization via channel response. ACM Computing Surveys (CSUR) 46(2), 25:1-25:32 (2013)

24. Youssef, M., Agrawala, A.: The horus wlan location determination system. In: Proceedings of the 3rd International Conference on Mobile Systems, Applications and Services (MobiSys). pp. 205-218. ACM, New York, NY, USA (2005) 